\title{
Collective Transport of Alpha Particles due to Alfvén Wave Instability
}

\author{
B.N. Breizman, H.L. Berk, and H. Ye \\ Institute for Fusion Studies \\ The University of Texas at Austin \\ Austin, Texas 78712
}

$\mathrm{DOE} / \mathrm{ET} / 53088--591$

DE93 009578

\begin{abstract}
Recently a new point of view has developed for describing saturation of discrete modes excited by weak sources. The method applies to the evolution of energetic particles in the beam plasma instability as well as to the description of how alpha particles evolve when they destabilize Alfvén waves under reactor conditions. Over a wide range of parameters the system produces pulsations, where there are relatively brief bursts of wave energy separated by longer intervals of quiescence. There are two types of pulsations; benign and explosive. In the benign phase, valid when particle motion is not stochastic, the distribution function is close to that predicted by classical transport theory, and the instability saturates when the wave trapping frequency equals the expected linear growth rate. If the field amplitude in a burst reaches the level where orbit stochasticity occurs, the quasilinear diffusion causes rapid transfer of particle energy to wave energy and rapid flattening of the particle distribution function. The bursting phase is followed by a relatively long quiescent time interval where the source provides the necessary free energy to regenerate the cycle. The critical issue is whether the instability develops to a high enough level to produce stochastic diffusion. In general this question can be assessed by using mapping methods to obtain criteria of
\end{abstract}

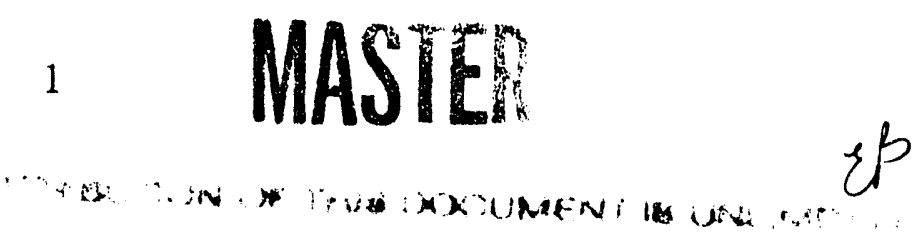


overlapping of orbit resonances. If overlap occurs, then the modes will saturate at a high level which will result in significant anomalous transport effects. This picture is consistent with recent observations of energetic beam losses in TFTR and D-III D due to the TAE mode. 


\section{Introduction}

The aim of this paper is to discuss several physics issues related to the problem of alpha particle confinement in an ignited tokamak in the presence of Alfvén wave excitations. We concentrate on the qualitative aspects of the problem, which are interesting in themselves as they have applications to problems in other areas. Our intention is to exhibit how a quantitative method of analysis can be developed to describe the nonlinear evolution of an instability driven by energetic particles together with the resulting anomalous diffusion of these particles. Though we have in mind Alfvén wave excitation by alpha particles, we will actually illustrate the mechanism of mode saturation by discussing the bump-on-tail instability, the simplest example of the effect we wish to describe. An appropriate model incorporates the following essential aspects of the problem: the discreteness of the mode spectrum, damping of waves from dissipation which arises from the background plasma, the presence of a particle source, and the effect of collisions which bring particles in or out of the resonant region where particles are interacting with waves.

The key concept in this discussion will be "resonant wave-particle interaction." Therefore, we will start with brief remarks on the pattern of resonances for alpha-particles with Alfvén waves and on how it can affect alpha-particle confinement. We will then use the model of the bump-on-tail instability to describe various nonlinear scenarios for the evolution of unstable modes. A single-mode regime will be considered first, followed by the case of many modes that can overlap and cause quasilinear diffusion. An important result of this part of the paper is the prediction of nonlinear bursts in the wave energy and in anomalous particle

loss. In the second part of the paper, we will indicate how a map model can be developed to study the interaction of energetic particles with Alfvén modes in a tokamak. Using this map, we calculate the threshold of particle stochastic diffusion. If generalized to incorporate wave 
dynamics, the map model can provide a self-consistent description of both mode saturation and resonant particle transport.

\section{Alpha Particle Losses due to Alfvén Resonances}

In a burning $\mathrm{D}-\mathrm{T}$ plasma, the alpha particles produced by fusion reactions have an initial energy of $3.5 \mathrm{MeV}$ with an isotropic angular distribution. These energetic particles slow down collisionally, mostly due to drag from electrons. As a result of the balance between the source and the drag, a stationary distribution of alpha particles is established with most of the alpha particle energy being transferred to the plasma.

This simple classical picture can change and anomalous losses can arise when alpha particles excite Alfvén modes. When both plasma and magnetic field are uniform, modes are excited by the particles which satisfy the resonant condition

$$
\omega-k_{\|} v_{\|}=0
$$

or

$$
v_{\|}=v_{A}
$$

where $v_{A}$ is the Alfvén velocity. In this case, the particles with initial parallel velocities below $v_{A}$ do not interact with the mode. These particles transfer their total energy to the plasma as drag brings the speed all the way down to $v=0$ in the shaded area 1 in Fig. 1 . The particles with larger parallel velocities must pass through the shaded area 2 before they it the resonance. Regardless of whether or not these particles are lost due to their interaction with the mode, they still release part of their energy as they pass through area 2 . With the pessimistic assumption that the particles are lost immediately when they hit the resonance, one can easily calculate the efficiency of alpha particle energy deposition:

$$
\eta \equiv \frac{\text { deposited energy }}{\text { initial energy }}=1-\left(\frac{v_{A}}{v_{0}}\right)+\left(\frac{v_{A}}{v_{0}}\right)^{2} .
$$


Here, $v_{0}\left(v_{0}>v_{A}\right)$ is the initial velocity of the alpha particles. Even though this "guaranteed" efficiency is always larger than $75 \%$, which may be tolerable in terms of energy balance, the anomalous losses can still be very important for a reactor since the wall loading by energetic particles could degrade the containment vessel.

Our $25 \%$ loss estimate is rather rough because the actual pattern of the resonances in a tokamak differs from the idealized picture shown in Fig. 1. In a toroidal geometry, resonance condition (1) changes to

$$
\omega-n \omega_{\varphi}+m \omega_{\theta}=0
$$

where $\omega$ is the mode frequency, $\omega_{\varphi}$ and $\omega_{\theta}$ are the frequencies related to the "toroidal" and "poloidal" motions of the particle, and $n$ and $m$ are integers. The resonance response of the alpha particles to the Toroidal Alfvén Eigenmode is illustrated by Fig. 2. This figure presents results of numerical simulations performed by C.T. Hsu using the actual mode structure and guiding center orbits in a tokamak. It is interesting to observe that there are resonances for both passing and trapped particles. By numerically finding the resonances and the collisional flow of the alpha particles in the phase space, one can generalize Eq. (3) and evaluate the upper limit of the anomalous losses of the alpha particles for a particular device. It is even conceivable that a proper choice of the magnetic configuration and plasma profile can make this upper limit tolerable for a reactor. These calculations are now under development. Another side of the problem, the one we concentrate on in this paper, is the analysis of the nonlinear particle and wave dynamics associated with the resonances.

\section{Saturation of a Single Plasma Wave with Particle Source and Particle Annihilation}

The problem we formulate in this section has much in common with the problem of nonlinear damping of a finite amplitude wave studied by $\mathrm{O}^{\prime} \mathrm{Neil}^{1}$ and Mazitov. ${ }^{2}$ However our problem is somewhat different in that it involves a particle source and sink which tend to establish 
an unstable distribution function in the region of phase space where resonance with a wave occurs. Part of this problem was treated by Mikhailovskii and Pyatak. ${ }^{3}$

The principal nonlinear effect in this problem is a modification of the distribution of resonant particles produced by the finite amplitude wave. This wave-particle interaction determines an energy transfer fivin particles to the wave. The wave itself is assumed to have the linear eigenmode structure. The tendency for the wave to flatten the resonant particle distribution ultimately weakens the instability drive and allows the background dissipation mechanisms to compete with the drive to determine the saturation level.

To proceed with the formal analysis, we consider a one-dimensional electrostatic wave with a potential $\varphi=\varphi_{0} \cos (k x-\omega t)$. In addition to the background plasma we inject a hot species with an injection velocity distribution $Q(v)$. These particles are assumed to annihilate at a rate $\nu_{a}$. The kinetic equation for the distribution function, $F$, is

$$
\frac{\partial F}{\partial t}+v \frac{\partial F}{\partial x}+\frac{q k}{m} \varphi_{0} \sin (k x-\omega t) \frac{\partial F}{\partial v}=-\nu_{a}(v) F+Q(v) .
$$

The wave-to-particle power transfer is given by

$$
\mathcal{P}=\frac{k \omega}{2 \pi} \varphi_{0} q \int_{0}^{2 \pi / k} d x \int d v \sin (k x-\omega t)\left(1+\frac{k u}{\omega}\right) F
$$

with $v=\omega / k+u$. The contribution to $\mathcal{P}$ is from a narrow region in velocity space so that $|k u / \omega| \ll 1$.

In linear theory we have the expression

$$
\mathcal{P}_{L}=\left.\frac{-q^{2} \pi}{2 m} \varphi_{0}^{2} \frac{\omega k}{|k|} \frac{\partial}{\partial v}\left(\frac{Q(v)}{\nu_{a}(v)}\right)\right|_{v=\omega / k}
$$

which corresponds to Landau damping $\left(\mathcal{P}_{L}>0\right)$, or growth $\left(\mathcal{P}_{L}<0\right)$, depending on the slope of the unperturbed distribution function.

For the nonlinear problem we assume that $F=F(\psi, u)$, with $\psi=k x-\omega t$, and $u=$ $v-\omega / k$. We also assume $\nu_{a} / \omega_{b} \ll 1$, where $\omega_{b}=\left(q k^{2} \varphi_{0} / m\right)^{1 / 2}$ is the trapped particle radian 
bounce frequency. Note that $\omega_{b}$ is also an estimate of the spatially periodic transit frequency of passing particles that are near the separatrix.

We then introduce the energy $E$ in the wave frame

$$
\frac{u^{2}}{2}+\frac{q \varphi_{0}}{m} \cos \psi=E
$$

and transfer from $u$ to $E$. By expanding $F$ in powers of $\nu_{a} / \omega_{b}$ we obtain the equations

$$
\begin{gathered}
\pm k \frac{\partial F_{0}^{ \pm}}{\partial \psi}=0 \\
\pm k \frac{\partial F_{1}^{ \pm}}{\partial \psi}=\frac{-\nu_{a}\left(\frac{\omega}{k}\right) F_{0}^{ \pm}+Q\left(\frac{\omega}{k}\right)}{u(E, \psi)} \pm\left[-\nu_{a}^{\prime}\left(\frac{\omega}{k}\right) F_{0}^{ \pm}+Q^{\prime}\left(\frac{\omega}{k}\right)\right]
\end{gathered}
$$

with \pm sign referring to positive and negative values of $u$.

It is shown in Ref. 4 that the solution of Eqs. (9) and (10) for passing and trapped particles results in the following expression for the nonlinear power transfer $\mathcal{P}_{\mathrm{NL}}$ :

$$
\mathcal{P}_{\mathrm{NL}}=-\left.2.18 \frac{\sqrt{2} q^{2} \omega \varphi_{0}^{2}}{m} \frac{k}{|k|} \frac{\partial}{\partial v}\left(\frac{Q(v)}{\nu_{a}(v)}\right)\right|_{v=\omega / k} .
$$

The ratio of $\mathcal{P}_{\mathrm{NL}} / \mathcal{P}_{L}$ is

$$
\frac{\mathcal{P}_{\mathrm{NL}}}{\mathcal{P}_{L}}=1.9 \frac{\nu_{a}}{\omega_{b}}
$$

Suppose a positive background dissipative power transfer, $\mathcal{P}_{d}$, is present with $-\mathcal{P}_{L}>\mathcal{P}_{d}$, so that there is linear instability. Then saturation arises when $\mathcal{P}_{\mathrm{NL}}=\mathcal{P}_{d}$ or when the wave reaches the level

$$
\omega_{b}=\left(\frac{q \varphi_{0} k^{2}}{m}\right)^{1 / 2}=1.9 \nu_{a}\left(\frac{\mathcal{P}_{L}}{\mathcal{P}_{d}}\right)=1.9 \nu_{a} \frac{\gamma_{L}}{\gamma_{d}}
$$

where $\gamma_{L}$ is the linear growth rate associated with the unperturbed distribution function

$$
F=\frac{Q(v)}{\nu_{a}(v)}
$$

and $\gamma_{d}$ is the dissipation rate caused by the background plasma.

It follows from Eq. (13) that, for $\gamma_{d}<\nu_{a}$, the source pumps the wave to an amplitude that gives a bounce frequency higher than the linear growth rate. As we will see in the 
discussion in Section 4, this is actually a necessary condition for the stability of the steadystate nonlinear solution.

\section{Pulsations of Isolated Modes}

We now consider the case when $\gamma_{d} \gg \nu_{a}$, i.e. the bounce frequency predicted by Eq. (13) is lower than $\gamma_{L}$, and show that in this case the above steady-state solution is unstable, basically to the same linear instability that exists in the unperturbed state. ${ }^{5}$ This observation readily follows from closely examining the response of linear theory. The linear growth rate, $\gamma_{L}$, is given by the following expression:

$$
\gamma_{L}=\frac{-2 \omega \pi e^{2}}{|k| m} \operatorname{Im} \int d v \frac{1}{\omega-k v} \frac{\partial F}{\partial v} .
$$

For a smooth distribution function formed in the absence of nonlinear waves, $\gamma_{L}$ reduces to

$$
\gamma_{L}=\frac{2 \omega \pi^{2} e^{2}}{|k| m} \int d v \frac{\partial F}{\partial v} \delta(\omega-k v) .
$$

In the case $\nu_{a} \ll \gamma_{d}$, the nonlinear distribution function found in Ref. 4 only differs from the unperturbed one in a small resonance region where particles are trapped in the wave. There the distribution is flattened over an area

$$
\delta v \approx \omega_{b} / k \equiv v_{b}
$$

Outside this region virtually the same $F$ is obtained as in the unperturbed case. Hence, if one attempts to evaluate $\gamma_{L}(\omega)$ in Eq. (15), with this locally flattened distribution function, one finds that, though $\gamma_{L}\left(\omega_{0}\right) \rightarrow 0$ with $\omega_{0}$ the real frequency of the background oscillation, when $\omega$ is allowed to be complex, the value for $\gamma$ is hardly changed from the value $\gamma_{L}$ found in the smooth case (the difference is $\mathcal{O}\left(\omega_{b} / \gamma_{L}\right)$ ). Hence the steady-state solution is unstable for sufficiently large $\gamma_{d}$, viz., $\gamma_{d} \gg \nu_{a}$.

This result indicates that the nonlinear response in the $\gamma_{d} \gg \nu_{a}$ limit cannot be a steady state. Instead a pulsation scenario is appropriate. Suppose the linear bump-on-tail 
instability with the smooth $F$ distribution develops at the rate $\gamma_{L}$. The distribution function would initially look like the thick solid line in Fig. 3, just when instability begins. Then, as basic and straightforward arguments indicate, the wave amplitude will grow until the bounce frequency of the trapped particles reaches the linear growth rate $\gamma_{L}$. The wave flattens the distribution function in the resonant region, which destroys the resonant particle drive in the manner described by $\mathrm{O}^{\prime} \mathrm{Neil}^{1}$ and Mazitov, ${ }^{2}$ and it is depicted by the thin solid curve in Fig. 3. However, with background dissipation present, this wave will now damp according to the equation $d W E / d t=-2 \gamma_{d} W E$ where $W E$ is the energy of the wave. Simultaneously, the source and the annihilation attempt to reconstitute the unstable distribution function in the flattened region, $\delta v / v \approx \omega_{b} / \omega \approx \gamma_{L} / \omega$, at a rate $\nu_{a}$. Thus the time for the wave energy to disappear is $1 / \gamma_{d}$, while the time for reconstitution is $1 / \nu_{a}$. After a time $1 / \nu_{a}$ the distribution is again ready to excite waves to an amplitude where $\omega_{b} \sim \gamma_{L}$. During intermediate times $1 / \gamma_{d}<t<1 / \nu_{a}$, precursor instability may arise, for example when the distribution is shaped like the dashed curve in Fig. 3. Low amplitude saturation will then occur due to particle trapping with a trapping frequency $\omega_{b 1} \approx \gamma_{L} \nu_{a} t<\gamma_{L}$. However these precursor waves do not destroy the free energy of the distribution in the velocity range

$$
\frac{\omega_{b 1}}{k}<\left|v-\frac{\omega}{k}\right|<\frac{\gamma_{L}}{k}
$$

Thus, low level precursor waves are expected prior to the largest "crash." After the largest crash, when $\omega_{b} \approx \gamma_{L}$, the distribution is again flattened over the interval $\delta v \approx\left|\delta v_{b}\right|$, with $\delta v_{b} \sim \gamma_{L} / k$, and then the process described repeats itself with an overall period. $\nu_{a}^{-1}$.

The need for a pulsation scenario can also be explained in terms of energy balance, which shows that it is energetically impossible to sustain a steady excitation level if $\nu_{a}<\gamma_{d}$. Over a long time scale, the average background dissipation can be estimated as $\gamma_{d} \overline{W E}$, with $\overline{W E}$ the time-averaged wave energy. This dissipation must be balanced by the free energy that is brought to the resonant region by the source and the annihilation. In a time $1 / \nu_{a}$ the 
free energy of the particles is built up and then converted to the maximum wave energy $W E_{\max }$ determined from the condition $\omega_{b} \approx \gamma_{L}$. This free energy comes from the particle distribution and is equal to the difference in kinetic energy in the distributions (a) and (c) in Fig. 3. Hence the estimate for the feed power into the wave is $\nu_{a} W E_{\max }$. Equating the feed power to the average dissipative power gives

$$
\overline{W E}=\left(\frac{\nu_{a}}{\gamma_{d}}\right) W E_{\max } .
$$

Since $\nu_{a}$ is assumed to be much less than $\gamma_{d}$, the average wave energy is much less than the maximum. Such a condition can only be achieved with relaxation oscillations, as depicted in the solid curves in Fig. 4. In contrast, for $\nu_{a} / \gamma_{d}>1$, as discussed above in Section 3, the wave energy saturates at a stationary level $W E^{*}=\left(\nu_{a} / \gamma_{d}\right) W E_{\max }$, as depicted by the dashed line of Fig. 4.

The pulsation description also applies when other classical transport processes are present besides particle annihilation. Rather than $\nu_{a}$, one in general case has $\nu_{\text {eff }}$. For pitch angle scattering, $\nu_{\text {eff }} \approx \nu \omega^{2} / \omega_{b}^{2}$ where $\nu$ is the $90^{\circ}$ scattering rate.

\section{Multiple Modes and Phase Space Explosion}

It follows from the previous section that a single unstable mode can only modify the particle distribution function locally. A different picture may arise when there are many unstable modes in the system with the fluctuation level exceeding the stochasticity threshold. 5,6 This is because particles now really do diffuse in phase space as there are no longer barriers to maintain an overall "inverted population" in the vicinity of the resonance region. This regime of the bump-on-tail instability is illustrated in Fig. 5. Below the critical amplitudes for mode overlap, the situation is depicted in Fig. $5 \mathrm{a}$, where the distribution flattens locally in the shaded regions, with an energy release proportional to $N \lesssim \frac{\omega}{\gamma_{L}}$, the number of modes. The picture changes drastically, as shown in Fig. 5b, when the resonances overlap.. Then all 
the free energy of the inverted gradient is available to pump the waves to yet higher levels, and to cause strong particle diffusion.

When the amplitudes of excited modes exceed the threshold of resonance overlap, the effect of the waves on the particles is described as quasilinear diffusion. ${ }^{7,8}$ The corresponding diffusion equation for the particle distribution function then has the form:

$$
\frac{\partial F}{\partial t}=\frac{\partial}{\partial v} D \frac{\partial F}{\partial v}-\nu_{a} F+Q(v)
$$

Here, the diffusion coefficient, $D(v)$, is related to the spectral density of the wave energy, $W(k)$, by

$$
D(v)=\frac{4 \pi^{2} e^{2}}{m^{2} v} W\left(\omega_{p} / v\right) .
$$

The function $W(k)$ is normalized by

$$
\int W(k) d k=U
$$

where $U$ is the wave energy per unit volume. The second and third terms on the right-hand side of Eq. (19) describe the source and the annihilation of the fast particles. We choose the source, $Q(v)$, and the annihilation rate, $\nu_{a}$, to meet the requirement that the "classical" stationary solution (14) of Eq. (19) has a sufficiently large positive derivative $\partial F / \partial v$ to drive the bump-on-tail instability in the presence of background damping. In order to simulate the feature that all potentially unstable modes are in a certain interval of phase velocities ranging from $v_{\min }$ tc $v_{\max }$, we set $D=0$ outside this interval.

We now add to Eq. (19) the equation for the evolution of wave energy,

$$
\frac{\partial W(k)}{\partial t}=2 \gamma W(k)-2 \gamma_{d} W(k)
$$

where

$$
\gamma=\frac{2 \pi^{2} e^{2} \omega_{p}}{k^{2} m} \frac{\partial F}{\partial v}\left(v=\omega_{p} / k\right)
$$


describes the wave excitation by energetic particles, while the second term takes into account background damping. The damping rate, $\gamma_{d}$, is assumed to be much less than the typical linear growth rate produced by the unperturbed stationary distribution (14) in the interval $\left(v_{\min }, v_{\max }\right)$. Thus the distribution (14) is strongly unstable. The stable stationary solution of Eqs. (19) and (22) differs due to quasilinear diffusion from Eq. (14) and is determined from the following equations:

$$
\begin{aligned}
& \frac{2 \pi^{2} e^{2} v^{2}}{m \omega_{p}} \frac{\partial F}{\partial v}-\gamma_{d}=0 \\
& \frac{\partial}{\partial v} D \frac{\partial F}{\partial v}-\nu_{a} F+Q(v)=0 .
\end{aligned}
$$

By integrating Eq. (23), $F(v)$ is obtained to within a constant. This constant is found by integrating Eq. (24) from $v_{\min }-\varepsilon$ to $v_{\max }+\varepsilon$ with the boundary conditions $\left.D(v) \frac{\partial F}{\partial v}\right|_{v=v_{\max }+\varepsilon}=$ $\left.D(v) \frac{\partial F}{\partial v}\right|_{v=v_{\min }-\varepsilon}=0$, and we obtain

$$
\int_{v_{\min }}^{v_{\max }} \nu_{a} F d v=\int_{v_{\min }}^{v_{\max }} Q d v
$$

With this condition taken into account, the solution for $F(v)$ is

$$
F=F_{1}+F_{2}
$$

where

$$
\begin{gathered}
F_{1}=\frac{\int_{v_{\min }}^{v_{\max }} Q d v}{\int_{v_{\min }}^{v_{\max }} \nu_{a} d v} \\
F_{2}=\frac{m \omega_{p}}{2 \pi^{2} e^{2}} \frac{\int_{v_{\min }}^{v} \gamma_{d} \frac{d v}{v^{2}} \int_{v_{\min }}^{v_{\max }} \nu_{a} d v-\int_{v_{\min }}^{v_{\max }} \nu_{a} d v \int_{v_{\min }}^{v} \gamma_{d} \frac{d v_{1}}{v_{1}^{2}}}{\int_{v_{\min }}^{v_{\max }} \nu_{a} d v} .
\end{gathered}
$$

The ratio of $F_{1}$ to $F_{2}$ is roughly of the order of $\gamma_{L} / \gamma_{d}$ where $\gamma_{L}$ is the linear growth rate for the unstable "classical" distribution function (14). When $\gamma_{L}$ is assumed much larger than 
$\gamma_{d}$, one can neglect the velocity dependent contribution to $F$ and then the distribution is nearly constant,

$$
F=\frac{\int_{v_{\min }}^{v_{\max }} Q d v}{\int_{v_{\min }}^{v_{\max }} \nu_{a} d v}
$$

We now combine Eqs. (23), (24), and (20) to find

$$
W\left(\omega_{p} / v\right)=\frac{m v^{3}}{2 \omega_{p} \gamma_{d}} \int_{v_{\min }}^{v}\left(\nu_{a} F-Q\right) d v .
$$

For the simplified distribution (29) we obtain

$$
W\left(\omega_{p} / v\right)=\frac{m v^{3}}{2 \omega_{p}^{\prime} \gamma_{d}} \int_{r_{\max }}^{v}\left(\nu_{a} \frac{\int_{v_{\min }}^{v_{\max }} Q d v_{1}}{\int_{v_{\min }}^{v_{\max }} \nu_{a} d v_{1}}-Q\right) d v .
$$

This equation shows that in quasilinear theory the vave energy density $W$ scales linearly with $Q$. However, when the source is very weak, the wave energy is insufficient to provide for mode overlap and Eq. (31) is not applicable. In this case, most of the time, each unstable mode forms a separate island in the phase space and quasilinear diffusion really does not arise, since island-to-island transitions are strongly suppressed. The source will then build up the slope of the distribution function until overlap is achieved.

The overlap condition can be estimated as follows. Let $E_{i}$ be the electric field amplitude of the $i$-th discrete mode. Then the energy density of this single mode, $E_{i}^{2} / 8 \pi$, can be estimated as

$$
\frac{E_{i}^{2}}{8 \pi} \sim \frac{\omega_{p}}{v N} W
$$

where $N$ is the total number of modes. To overlap the neighboring resonances one needs

$$
\frac{\Delta v}{v}>\frac{1}{N}
$$

where $\Delta v$ is the velocity perturbation of the particle that resonates with the $i$-th mode. For $\Delta v$ we have

$$
\Delta v=\sqrt{\frac{e v E_{i}}{m \omega_{p}}} .
$$


By combining Eqs. (32)-(34) we find the following criterion of resonance overlapping:

$$
W>\omega_{p} \frac{m^{2} v^{2}}{8 \pi e^{2} N^{3}} .
$$

Taking into account Eq. (31) we rewrite Eq. (35) as a condition on the particle source for the validity of the steady-state quasilinear solution,

$$
Q>\frac{\gamma_{d} m \omega_{p}^{2}}{4 \pi e^{2} N^{3} v} \equiv Q_{c} .
$$

We then conclude that quasilinear stationary solution given by Eqs. (29) and (30) breaks down when the intensity of the source is below the critical value, $Q_{c}$, given by Eq. (36). The "classical" stationary solution (14) is also inappropriate since we have chosen it to be strongly unstable. This indicates again that the system does not reach a stationary state but rather creates bursts which explosively release the free energy built up by the particle source.

In order to estimate the energy of a burst, we first neglect the particle source and the wave damping. As long as the excited discrete modes do not overlap (Fig. 5a) each mode saturates when the bounce frequency of a resonant particle trapped by the mode reaches the linear growth rate $\gamma$. In this regime, one has

$$
\frac{\Delta v}{v}=\frac{\gamma}{\omega_{p}} .
$$

As time progresses the source causes the slope of the distribution to build up so that $\gamma$ increases and $\Delta v / v$ eventually reaches the value $1 / N$. At this critical value of $\gamma$, the total free energy of the unstable distribution becomes available for the burst (Fig. 5b). This energy can be estimated as the energy that is released through global flattening of the distribution with $\gamma=\gamma_{\text {crit }} \equiv \frac{\omega_{p}}{N}$ :

$$
U_{\text {burst }} \sim \frac{m^{2} \omega_{p e}^{2}}{24 \pi^{2} e^{2} N}\left(v_{\max }-v_{\min }\right)^{3} \frac{1}{v_{\max }+v_{\min }} .
$$


This consideration shows that a weak source is unable to build up a particle distribution with a free energy exceeding the value given by Eq. (38).

By comparing Eqs. (35) and (38) we may note that the wave energy required for mode overlapping is much less than $U_{\text {burst }}$ :

$$
U_{\text {overlap }} \sim \frac{U_{\text {burst }}}{N^{2}} .
$$

Therefore, the burst is well described by quasilinear theory. This theory predicts buildup of the wave energy and flattening of the particle distribution within a timescale $N / \omega_{p}$. Then the waves damp at a rate $\gamma_{d}$, while the distribution function remains flat since the source is too weak to change the particle distribution within the damping timescale. The third, longer phase, is the restoration of the free energy required for the next burst. The time interval, $\tau_{\mathbf{r s t}}$, of the restoration, is determined by the energy balance. Hence, $\tau_{\mathrm{rst}}$ is inversely proportional to the intensity of the source:

$$
\tau_{\text {rst }} \sim \frac{U_{\text {burst }}}{m Q v_{\max }^{3}} .
$$

It is interesting to note that when $\gamma_{\text {crit }} \ll \gamma_{L}$ the average power transfer from particles to waves is rather insensitive to whether the system reaches quasilinear stationary state or creates bursts. This result is straightforward to observe when one writes the average power transfer to the waves, $\bar{P}_{w}$, as a difference between the power supplied from the source and the dissipation from annihilation:

$$
\bar{P}_{w}=\frac{1}{T} \int_{0}^{T} d t \int_{v_{\min }}^{v_{\max }} \frac{m v^{2}}{2}\left(Q-\nu_{a} F\right) d v,
$$

where the averaging period, $T$, is over many burst periods. This expression only depends on the particle distribution function which, when $\gamma_{\text {crit }} \ll \gamma_{L}$, is close to plateau (27) in both cases. The bursts of the wave energy are obviously easier to observe than the corresponding small deviations of the particle distribution function from the plateau. It should also be noted 
that, most of the time between bursts, the distribution function is metastable. Therefore, if an appropriate triggering mechanism is available, the system bursts before the accumulated free energy reaches the critical value given by Eq. (38).

\section{Map Model for Alpha Particle Interaction with Toroidal Alfvén Modes}

In this section, we address the problem of when alpha particle motion in a tokamak becomes stochastic under the influence of Toroidal Alfvén Eigenmodes. The stochasticity threshold can be determined by studying the test particle motion in a given wave field. A similar approach has been used for studying the effects of magnetic field ripple ${ }^{9,10}$ and low-frequency perturbations. ${ }^{11}$ A procedure that allows for an analytic estimate, or rapid numerical calculation, is the reduction of the particle motion to a two-dimensional map. A detailed derivation of this map is given in Ref. 12. Here, we only remind the reader of some key elements used in Ref. 12 and present an example from our numerical results which illustrate the transition to stochasticity. As the wave amplitudes are sufficiently small, a single-transit alpha particle response to the linear mode can be calculated using linearized orbit theory. Longer time nonlinear dynamics of the alpha particles is then simulated by following the map for many transits.

The reduction to a map is made possible by a number of preliminary simplifications based on the physical nature of the problem. First, we consider only the passing particles in a large aspect ratio tokamak. Most of these particles are far enough from the trapped-passing boundary so that their unperturbed parallel and perpendicular velocities can be treated as constant. Second, we use the fact that it is the toroidal angular momentum, rather than the particle energy, that primarily changes during the alpha particle interaction with TAE modes. This means that variations in $v_{\|}$and $v_{\perp}$ are negligible even when a perturbation is present. Third, we take into account that there is an important range of parameters where 
the alpha particle response is insensitive to the finite Larmor radius effects, even if the scale length of the radial mode structure is comparable or smaller than the alpha particle Larmor radius.

With these simplifications, the following "effective" Hamiltonian was derived in Ref. 12 for the alpha particle motion in a poloidal cross-section:

$$
H\left(p_{\theta} ; \theta ; t\right)=\frac{e v_{\|}}{c R_{0}} \psi(r)-\frac{e}{c} B_{0} v_{d} r \cos \theta+e \sum_{p}\left[\left(1-\frac{v_{\|} \mathbf{b}}{i \omega_{p}} \cdot \nabla\right) \delta \phi_{p}+\text { c.c. }\right] \text {. }
$$

Canonical variables are poloidal angle $\theta$ and momentum $p_{\theta}$ related to minor radius $r$ by

$$
p_{\theta} \equiv \frac{e}{c} \frac{1}{2} B_{0} r^{2}
$$

where $B_{0}$ is the toroidal magnetic field on the magnetic axis. Other notations used in Eq. (42) are as follows: $R_{0}$ is the major radius of the magnetic axis,

$$
\psi(r) \equiv R_{0} \int_{0}^{r} B_{\theta}\left(r^{\prime}\right) d r^{\prime}
$$

is poloidal flux function, $v_{d} \equiv\left(v_{\|}^{2}+\frac{1}{2} v_{\perp}^{2}\right) / \Omega R_{0}$ is the alpha particle drift velocity, $\Omega \equiv e B_{0} / M c$ is the alpha particle gyrofrequency, $e$ and $M$ are the alpha particle charge and mass, $\mathbf{b} \equiv \mathbf{B} / B$ is the direction of local equilibrium magnetic field, $p$ is a mode index, $\delta \phi_{p}$ is the electrostatic potential of the perturbed field, $\omega_{p}$ is the frequency of mode $p$. The form of the perturbed fields is taken as

$$
\delta \mathbf{E}=-\nabla \delta \phi-\frac{1}{c} \partial_{t} \delta A_{\|} \mathbf{b}, \quad \delta \mathbf{B}=-\mathbf{b} \times \nabla \delta A_{\|}
$$

with

$$
\partial_{t} \delta A_{\|}=-\mathbf{c b} \cdot \nabla \delta \phi
$$

To treat the perturbation we use the following representation for the wave function of the $p$ th mode

$$
\delta \phi_{p}(r, \theta, \varphi, t) \equiv \sum_{m} \phi_{m}^{(p)}(r) e^{i\left(n \varphi-m \theta-\omega_{p} t\right)}
$$

Note that a given mode $p$ has a particular toroidal mode number $n$. 
With the Hamiltonian (42), the equations of motion are

$$
\dot{p}_{\theta}=-\frac{\partial H}{\partial \theta}, \quad \dot{\theta}=\frac{\partial H}{\partial p_{\theta}} .
$$

However, for the map model it is more convenient to use $\theta$ as the independent variable instead of $t$. With this conversion, $H$ and $t$ become the new canonical variables, and the new Hamiltonian is $p_{\theta}$.

The equations of motion in terms of $(H, t)$ are now given by

$$
\frac{d H}{d \theta}=-\frac{\partial p_{\theta}}{\partial t}, \quad \frac{d t}{d \theta}=\frac{\partial p_{\theta}}{\partial H} .
$$

Equations (42), (43) and (49) allow us to construct an area preserving map in a standard way, with a single step of the map being one transit in $\theta$ between $-\pi$ and $\pi$.

To apply the map to TAE modes ${ }^{13--15}$ one first has to specify the radial structure of the mode. To illustrate the capabilities of the model we use the following form of $\phi_{m}(r):^{15}$

$$
\phi_{m}(r)=\Phi_{m} \int_{r_{m}}^{r} \frac{\Delta_{m}+\alpha_{m}\left(r^{\prime}-r_{m}\right)}{\left(r^{\prime}-r_{m}\right)^{2}+\Delta_{m}^{2}} d r^{\prime}
$$

where $r_{m}$ is the gap location and $\Delta_{m}$ the "local mode width." Note that the map model itself is more general than expression (50) which has some restrictions in regard to applicability. The results can be improved by using the actual numerically determined mode structure.

Despite these simplifications, many important physical details are retained by the map. For example, the structure of the resonant interaction for a given particle depends on where the unperturbed orbit is located relative to the radial structure of the mode, and on the ratio of the orbit width to the mode width. Of particular interest is the case when the particle excursion (due to the guiding center drift) from the flux surface is comparable or even larger than the radial width of the mode structure. For example, in the linear theory ${ }^{16}$ that has been developed, which is closely related to our map model, it was shown that the instability drive is substantially reduced compared to what would be extrapolated using the thin-orbit 
theory. Along with the changes in the growth rate, new resonances appear when the orbit is thick; these resonances being negligible for the thin orbit case.

The resonances of the map and the nonlinear oscillations of the resonant orbits are described in Ref. 12. In that work a Chirikov criterion ${ }^{17}$ is formulated for the onset of stochasticity, by equating the resonance width to the distance between ajacent resonances. In terms of the amplitude of the perturbed radial magnetic field, the stochasticity threshold has the form: ${ }^{12}$

$$
\frac{\delta B_{r}}{B_{0}} \geq \frac{r_{m}}{64 m R_{0}} \frac{1}{q s},
$$

where $q$ and $s \equiv r q^{\prime} / q$ are the safety factor and the shear parameter at the mode location. For typical tokamak parameters, Eq. (51) gives the threshold $\delta B_{r} / B_{0} \approx 1.5 \times 10^{-3} / \mathrm{m}$. This result is in qualitative agreement with the earlier numerical integration results obtained by Sigmar et al. ${ }^{18}$

The transition from regular to stochastic motion, obtained with the numerical implementation of the map, is illustrated by Fig. 6 . This figure shows a poloidal cross section in which the mode location is depicted by the solid line. The crosses indicate the points where the orbit crosses the mode surface. The dotted lines depict inner and outer envelopes of the particle orbit. These two boundaries almost coinside when the perturbation is small (Fig. 6a), which means that alpha particle excursion from the equilibrium orbit is negligible. For a larger perturbation, (Fig. 6b) the orbit covers the whole area between the dotted lines. If more than one mode were present in the system, the particle could diffuse all the way to the boundary and eventually be lost. Ref. 12 presents additional numerical results obtained with this map and a discussion of how the map can be generalized to describe the self-consistent interaction of particles and waves. Thus time evolution of the waves depends on the history of the particle distribution function. 


\section{Conclusions}

In order to understand alpha particle transport arising from the excitation of Alfvén modes, we have considered mechanisms of nonlinear mode saturation and particle stochastic diffusion for a simple model of bump-on-tail instability. This model has important features that are in common with the alpha particle problem which include a weak source of energetic particles and wave damping from the background plasma. These features clearly demonstrate the likelihood that a bursting response characterizes the nonlinear behavior of the system.

Depending on the parameters, bursts can be either "benign" or explosive. Benign bursts only flatten the distribution function locally and waves do not tap the overall free energy source of the energetic particles. The waves grow only up to a natural saturation level where the bounce frequency of particles in the wave equals the linear growth rate. At this stage, the local free energy drive is saturated, and no further energy can be extracted from energetic particles by the wave. Subsequently, the wave damps due to background dissipation, and a time interval determined by classical transport processes needs to elapse before waves re-excite. As the resonant particles cannot move beyond the island boundary of the wave, the overall global distribution function is still close to the one predicted from the collisional transport theory.

Explosive bursts arise when the estimated level of wave saturation, given by the equality of the particle bounce frequency and linear growth rate, causes the resonances of neighboring modes to overlap. Now, when there is wave activity, particles really diffuse in phase space according to the predictions of quasilinear theory. For a sufficiently strong source the noise level is steady. However, with a weaker source the systern is quiescent most of the time. The particle distribution builds up from its flattened state until the stored free energy can cause saturated modes to reach the point of overlap. During the build-up of the distribution function there can be precursors, but they just lead to the benign saturation of the waves 
previously described, with the overall increase in the distribution function continuing as if there were no oscillations. However, near the point of criticality, typically determined by mode overlap, the distribution will "explode," and again relax to the flattened quasilinear state, where the cycle repeats. Sometimes the critical point can be determined by other trigger mechanisms.

The application of this picture to more complicated problems, such as Alfvén instabilities, is clear. In the Alfvén problem, the classical transport mechanisms involve drag and pitch angle scattering in velocity space, while the quasilinear relaxation primarily involves spatial diffusion. The phase space explosions then imply rapid radial diffusion, which can lead to direct and rapid energetic particle loss to the plasma edge. Such an interpretation is quite compatible with experimental observations. ${ }^{19,20}$ Specific predictions as to how alpha particles evolve for a given case will require determining the detailed instability growth rates for the mode spectrum as well as analyzing the mechanisms for particle resonance.

To study alpha particle diffusion caused by Alfvén modes we have developed a map model which describes the nonlinear interaction between the alpha particles and toroidal Alfven waves, in particular the TAE modes. With this model we have obtained an analytic expression for the critical wave amplitude for the onset of particle orbit stochasticity and verified this estimate numerically, both are essential quantities for assessing the alpha particle confinement in a fusion tokamak.

Using the stochasticity threshold and the estimates for the saturation level of a single TAE mode driven by alpha particles, we can formulate the condition under which the effect of the mode on alpha particle losses is expected to be insignificant. This is the case when the mode saturates below the stochastic threshold. It should be noted that the stochastisity does not necessarily cause diffusion over the entire profile. Global diffusion requires the presence of many modes with different radial positions over the poloidal cross section, and it may occur that diffusion is only in a limited region of space. Global diffusion is of course 
unfavorable for the confinement of the alpha particles. However, even this regime may still be acceptable because a substantial fraction of the alpha particle energy can still be absorbed by the plasma, since typically the resonance condition occures at energies below the birth energy. To get a better feeling about global diffusion we plan to study a self-consistent map which would incorporate wave dynamics.

\section{Acknowledgments}

This work was supported by the U.S. Department of Energy under contract No. DE-FG0580ET-53088. 


\section{References}

1. T.M. O'Neil, Phys. Fluids 8, 2255 (1968).

2. R.K. Mazitov, Zh. Prikl. Mekh. i Techn. Fiz. 1, 27 (1965).

3. A.B. Mikhailovskii and A.I. Pyatak, Sov. Phys. JETP 46, 721 (1977).

4. H.L. Berk and B.N. Breizman, Phys. Fluids B 2, 2226 (1990).

5. H.L. Berk, B.N. Breizman, and H. Ye, Phys. Rev. Lett. 68, 3563 (1992).

6. H.L. Berk and B.N. Breizman, "Scenarios for the Nonlinear Evolution of Beam-Driven Instability with a Weak Source," Presented at Stix Symposium, Institute for Fusion Studies Report \#582 (1992), to be published.

7. A.A. Vedenov, E.P. Velikhov, and R.Z. Sagdeev, Nucl. Fusion Suppl. 2, 465 (1962).

8. W.E. Drummond and D. Pines, Nucl. Fusion Suppl. 3, 1049 (1962).

9. R.J. Goldston, R. B. White, and A. H. Boozer, Phys. Rev. Lett. 47, 647 (1981).

10. S.V. Konovalov and S. V. Putvinskii, Fusion Technology 18, 397 (1990); S. V. Putvinskii, "Alpha Particles in a Tokamak," Voprosy Teorii Plasmy (Reviews of Plasma Physics), edited by B.B. Kadomtsev (Energoatomizdat, Moscow, 1990, in Russian), Vol.18, p.209.

11. H.E. Mynick, "Transport of Energetic Ions by Low- $n$ Magnetic Fluctuations," to be published in Phys. Fluids B (1993).

12. H.L. Berk, B.N. Breizman, and H. Ye, "Map Model for Nonlinear Alpha Particle Interaction with Toroidal Alfvén Waves," to be published in Phys. Fluids B (1993). 
13. C.Z. Cheng, L. Chen, and M.S. Chance, Ann. Phys. (N.Y.) 161, 21 (1985).

14. C.Z. Cheng and M.S. Chance, Phys. Fluids 29, 3695 (1986).

15. M.N. Rosenbluth, H.L. Berk, J.W. Van Dam, and D.M. Lindberg, Phys. Fluids B 4, $2189(1992)$.

16. H.L. Berk, B.N. Breizman, and H. Ye, Phys. Lett. A 162, 475 (1992).

17. B.V. Chirikov, Phys. Reports 52, 265 (1979).

18. D.J. Sigmar, C.T. Hsu, R. White, and C.Z. Cheng, Phys. Fluids B 4, 1506 (1992).

19. K.L. Wong, Phys. Rev. Lett. 66, 1874 (1991).

20. W.W. Heidbrink, E.J. Strait, E. Doyle, and R. Snider, Nucl. Fusion 31, 1635 (1991). 


\section{Figure Captions}

1. Collisional slowing down of the alpha particles in the presence of an Alfvén mode. The particles are injected at $v=v_{0}$ and lose their energy due to drag on electrons. In area 1, particles are not affected by the resonance. The particles from area 2 hit the resonance and then can possibly be lost before they deposit their remaining energy to the plasma.

2. Resonance response of alpha particles to the Toroidal Alfvén Eigenmode. The change in particle energy versus the pitch angle parameter is plotted for particles with an initial energy $3.5 \mathrm{MeV}$ and a given toroidal angular momentum; a-passing particle resonance, $b$-trapped particle resonance.

3. Time behavior of the bump-on-tail distribution function near the resonant mode phase velocity. The thick solid curve (a) indicates the distribution just before its relaxation; the thin solid curve (c) is just after relaxation; the dashed curve (b) is for an intermediate time when the distribution is being reconstituted.

4. Relaxation oscillations. If $\nu_{a}<\gamma_{d}$, relaxation oscillations arise as shown by solid curves. If $\nu_{a}>\gamma_{d}$, the wave energy saturates at a steady-state level $W E^{*}=\left(\nu_{a} / \gamma_{d}\right) W E_{\max }$.

5. Effect of resonance overlapping. In (a) modes do not overlap, and the relaxed distribution is just locally flattened, with the general shape of the inverted equilibrium distribution preserved. When modes overlap as in (b), the distribution flattens completely over the entire spectrum, with a much larger conversion of free energy to wave energy.

6. Regular and stochastic poloidal motion of an alpha particle interacting with a single TAE mode; (a) - regular motion at low mode amplitude $\delta B_{r} / B_{0}=10^{-4}$, (b) - stochastic 
motion at $\delta B_{r} / B_{0}=10^{-3}$. 


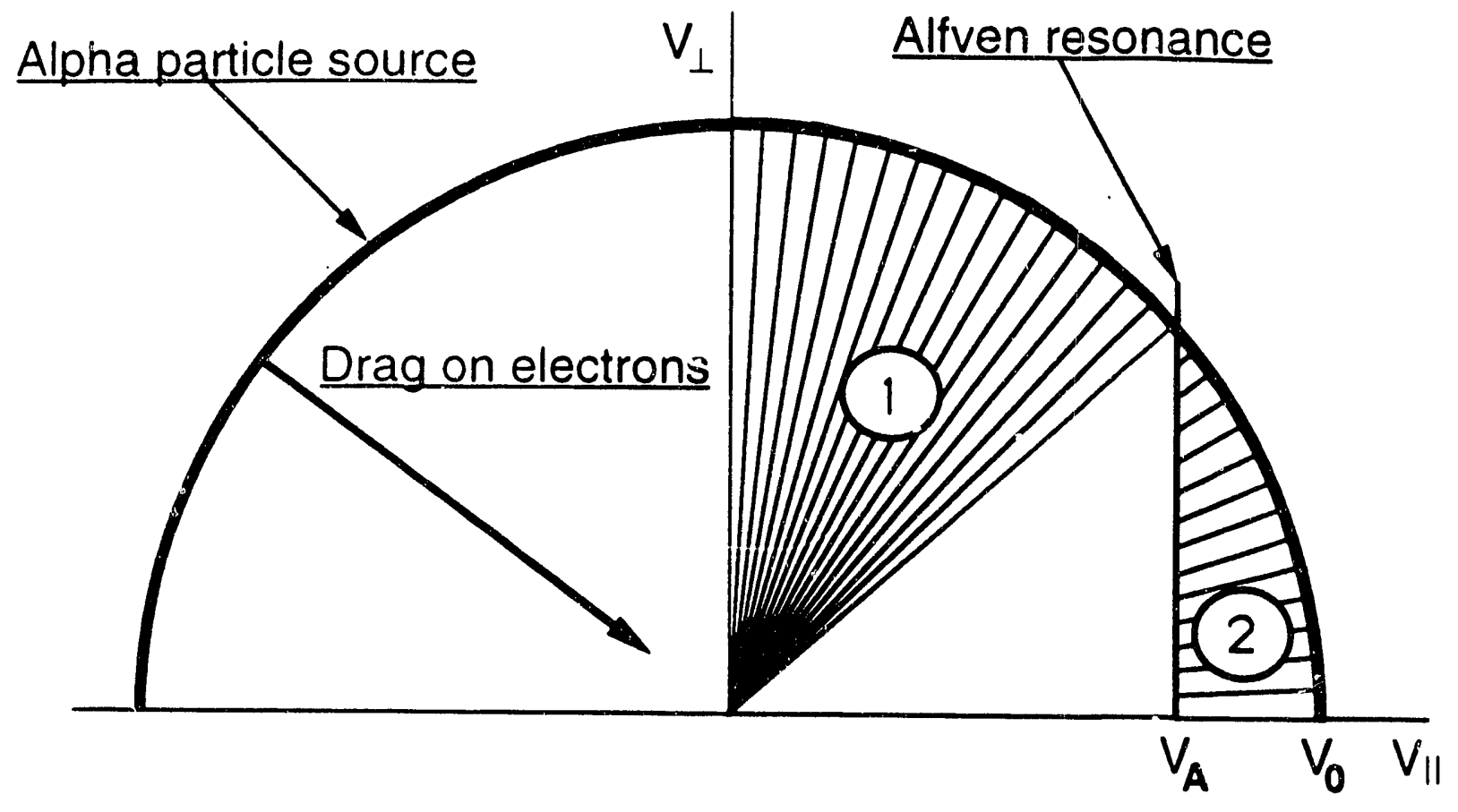

Figure 1 


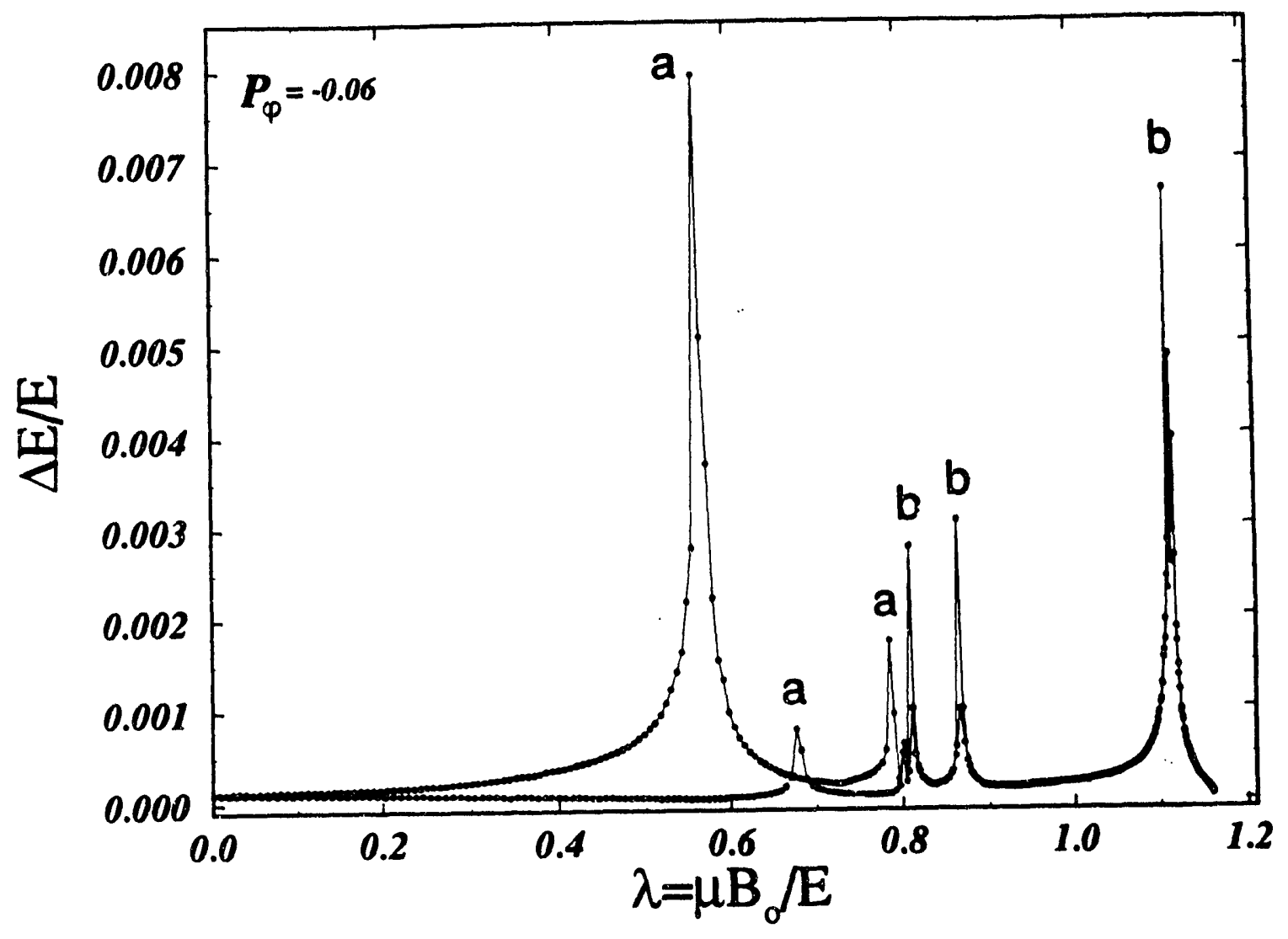

Figure 2 


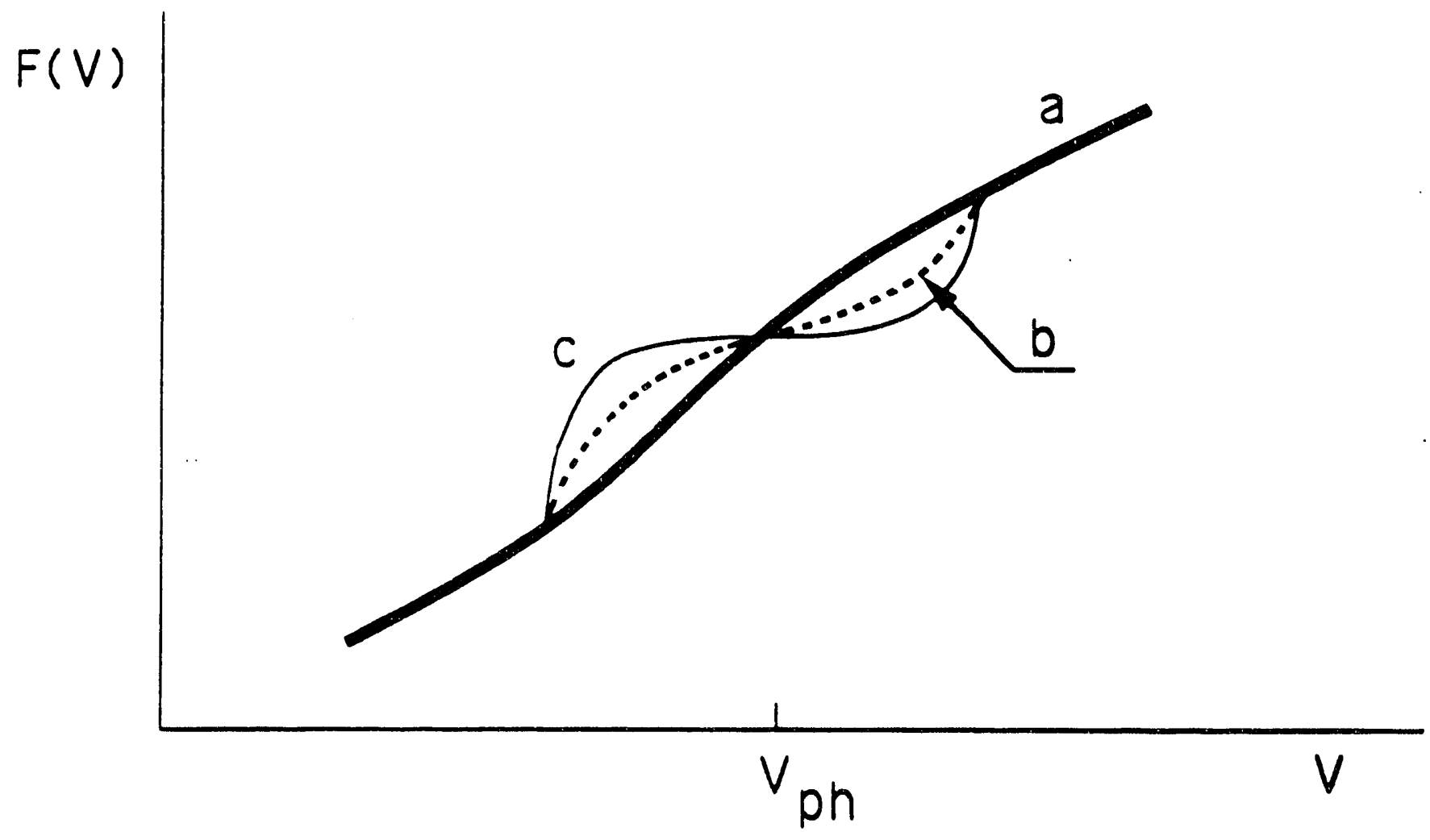

Figure 3 


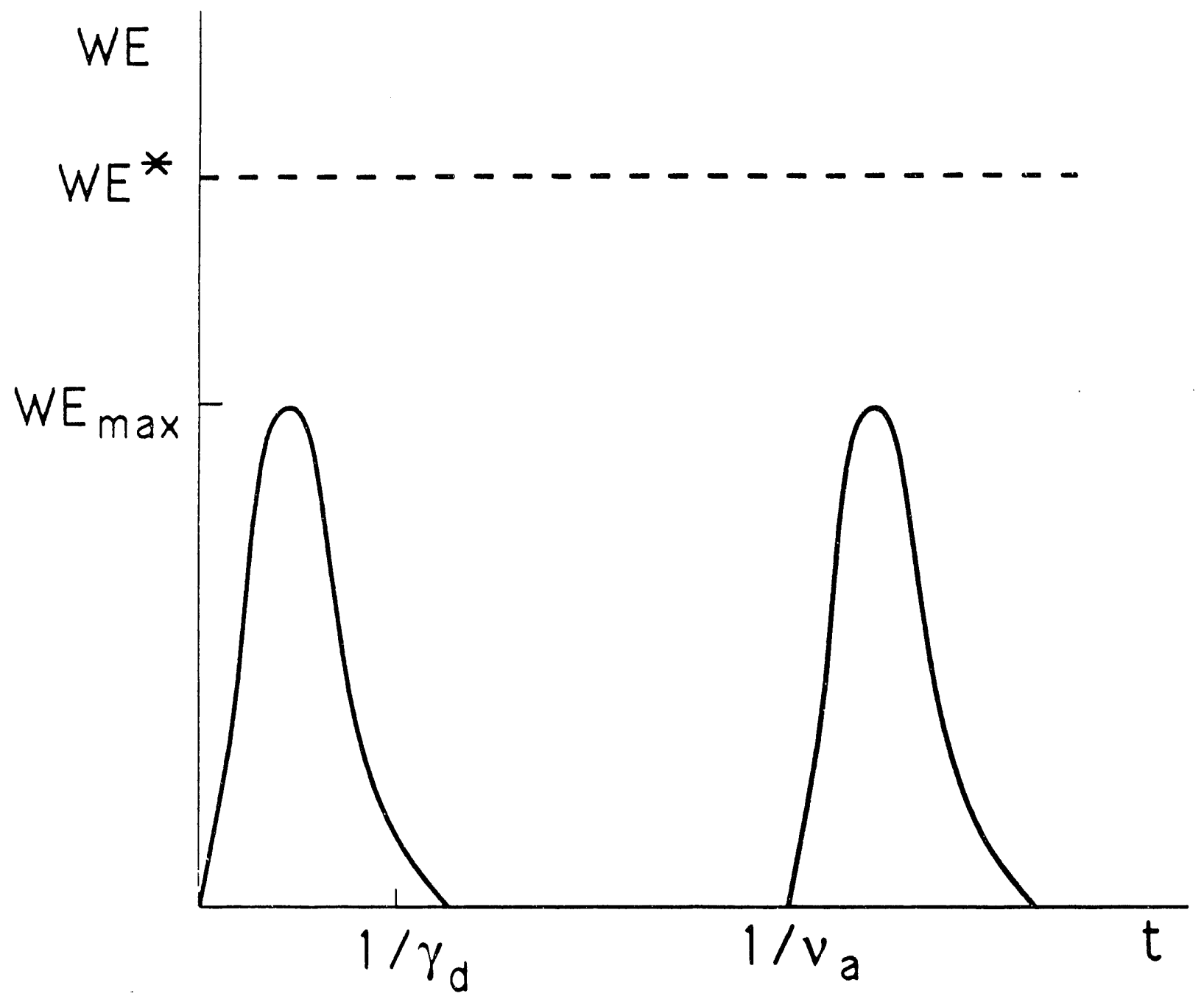

Figure 4 

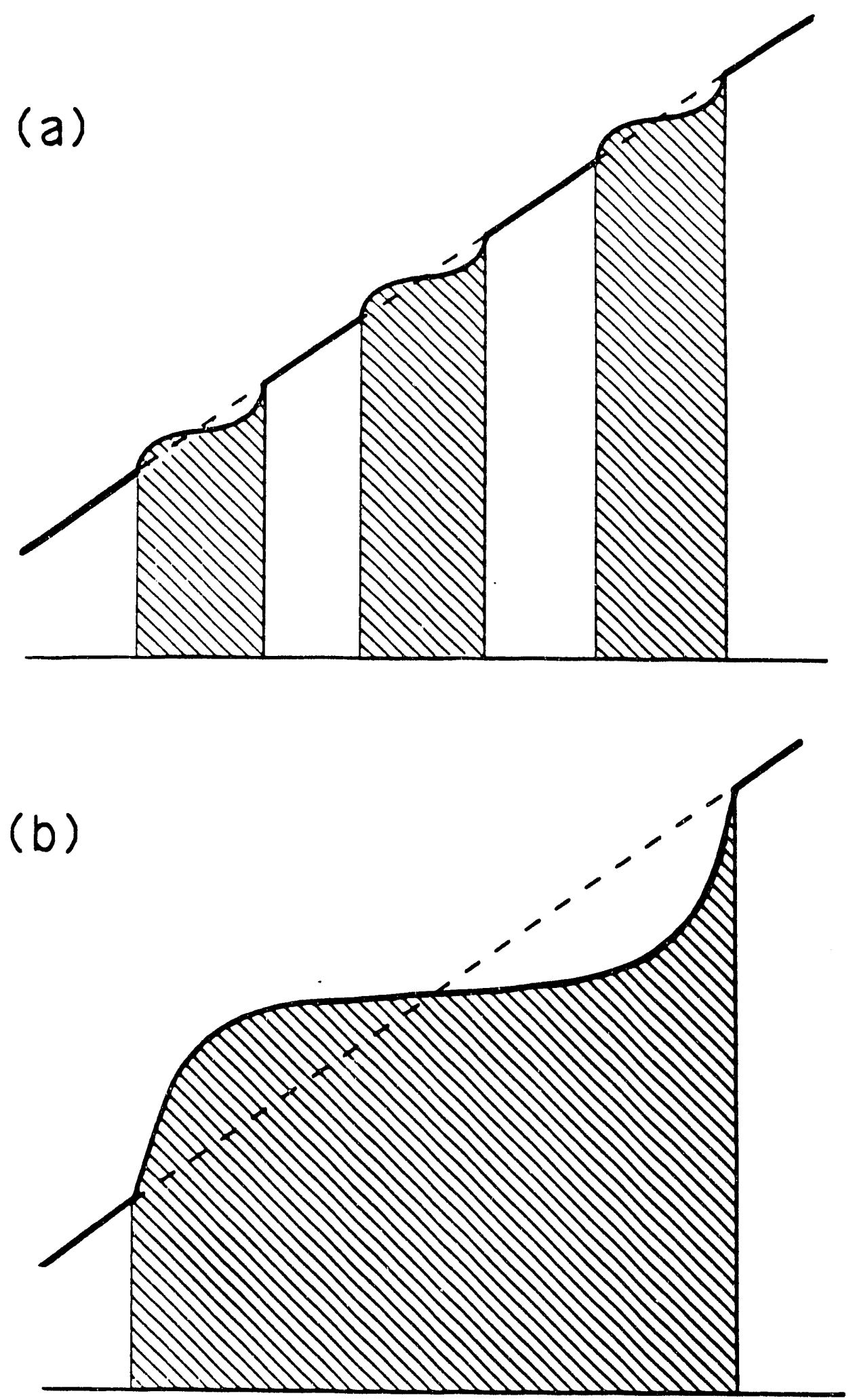

Figure 5 

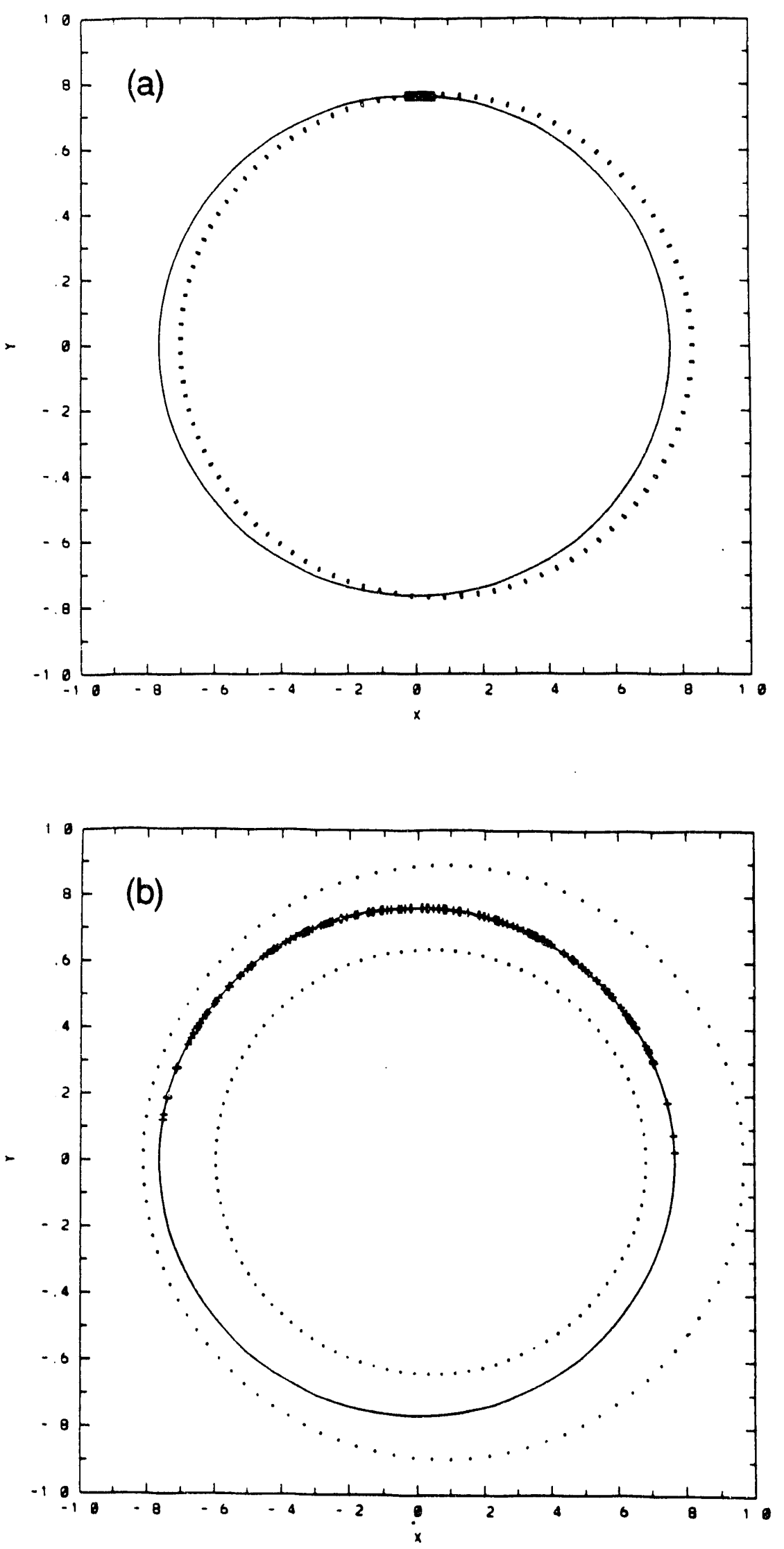

Figure 6 

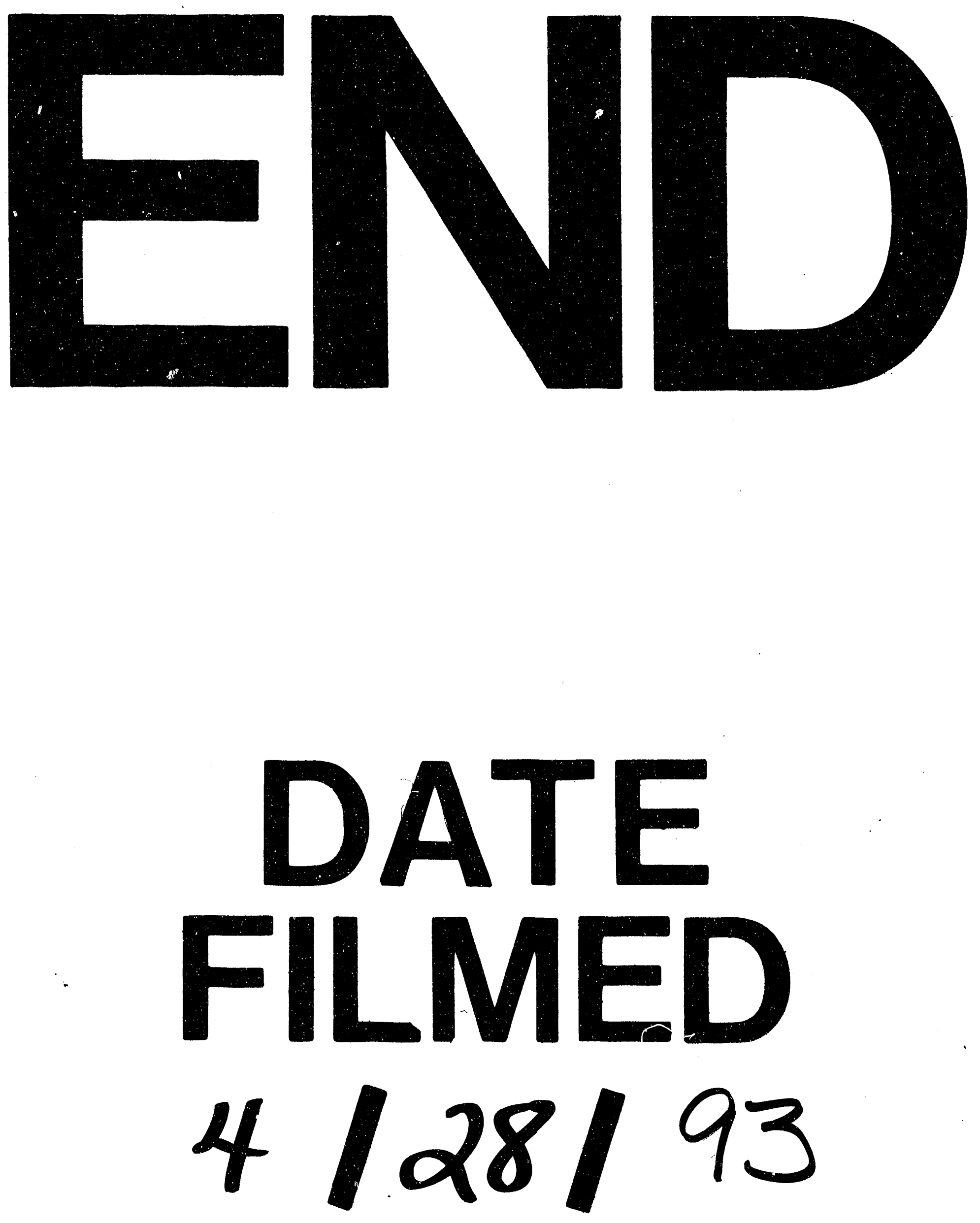
